\title{
Dislocation Analysis of Semiconductor Devices using 3D Rotation Imaging Technique of Dedicated STEM
}

Tae Sun Back, Soon Ju Lee, Jin Woo Jung, Jong Hyeop Kim and Ho Joung Kim

Research and Development Division, Hynix Semiconductor Inc., San 136-1, Ami-ri, Bubal-eub, Ichon-si, Kyoungki-do 467-701, Korea

We report the results of STEM 3D rotation imaging technique to analyze the crystalline defect due to the stress of dielectric material.

TEM samples were prepared at specific fail points by conventional focused ion beam (FIB) technique [1]. The dislocations on the edge of field oxide and abnormal diffraction contrast were observed by TEM (Fig.1). The existence of these dislocations couldn't explain the leakage failure mechanism; Therefore, we tried to analyze this abnormal diffraction contrast beneath the gate. But it was difficult to figure out whether it was a crystalline defect or not in $2 \mathrm{D}$ images.

Determining the nature of the abnormal diffraction contrast was difficult to figure out. To confirm this issue we made pillar type samples with 3D specimen holder for 3D observation [2-4]. In conclusion, we found the dislocation in the bottom of the gate corresponds to the electrical failure analysis data through the 3D rotation image observation (Fig. 2). In order to get confirm this issue, we fabricated specimen with the thin thickness enough to fill the gate and bit line contact as well as measured by STEM using 3D rotation imaging method at different rotation angles, such as 0, 90, and 180 degrees. The diffraction contrast revealed a screw dislocation connecting bottom dislocation with the gate. This analysis technique is an effective method to get at the root of physical failure in semiconductor devices.

\section{References}

[1] Joachim Loos, Jeroen K.J. van Duren, Francis Morrissey, Rene' A.J. Janssen, Polymer 43 (2002) 7493.

[2] Yaguchi, T., et al Proc 29th Int. Symp. for Testing and Failure Analysis, Santa Clara, CA, Nov. (2003) 282.

[3] Kamino, T. et al, Journal of Electron Microscopy 53 (5) (2004), 563.

[4] Aritoshi Sugimoto, Yukio Kembo, Kenji Watanabe, Ph. D., Toshie Yaguchi, Ph. D. Hitachi Review 55 (2006), 2. 

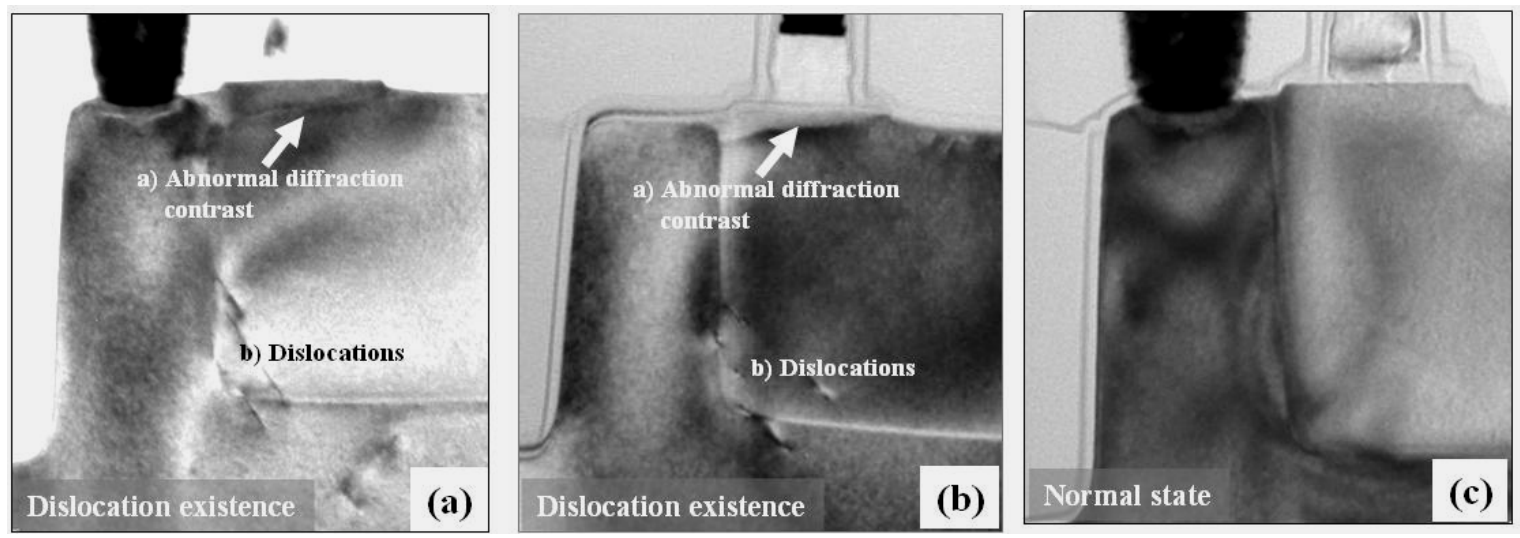

FIG. 1. TEM images of (a) 1st failure analysis, (b) 2nd failure analysis, (c) Normal state of the sample.
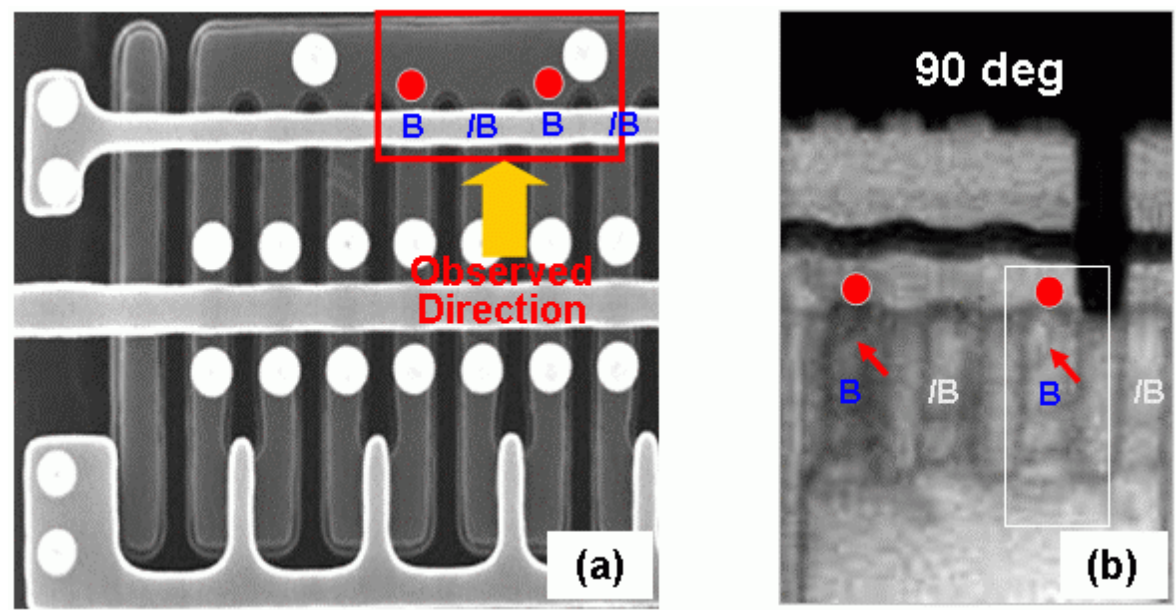

FIG. 2. (a) SEM image of observed direction, (b) STEM image of pillar type samples with 3D specimen holder for 3D observation.
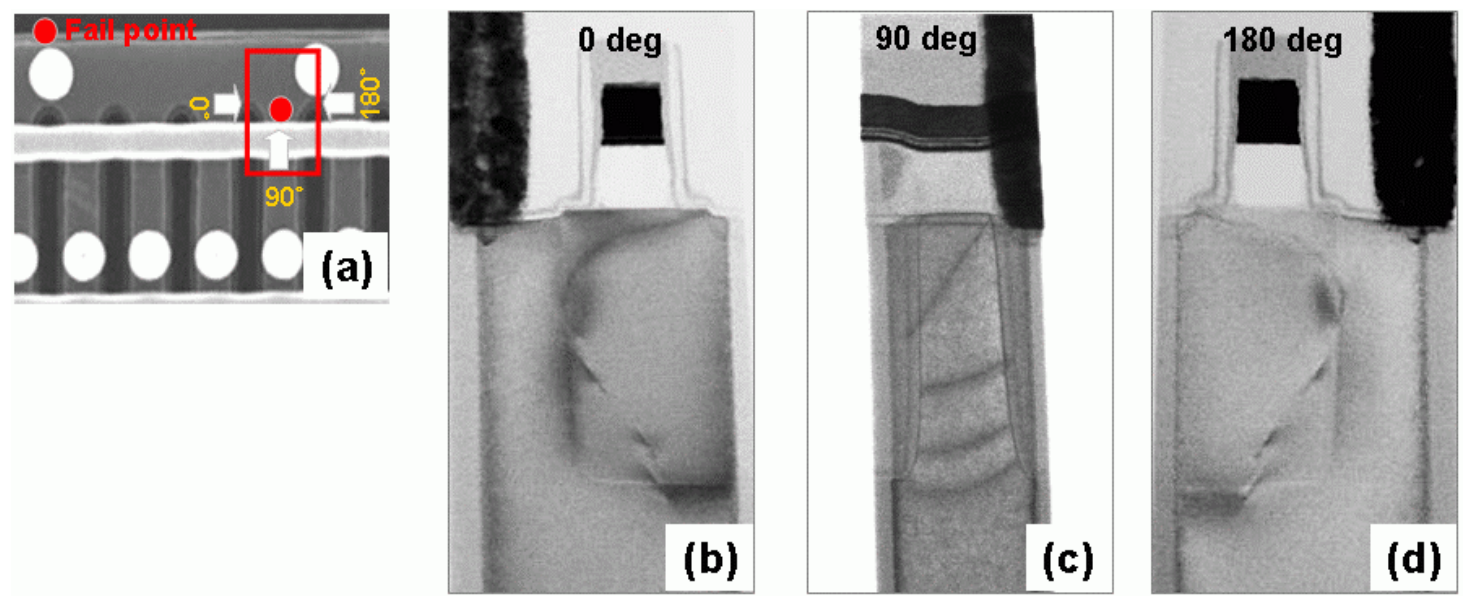

FIG. 3. (a) SEM image of observed direction, (b) TEM images of specimen with the thin thickness at different rotation angles with (b) 0 , (c) 90, (d) 180 degrees. 